\title{
Proteomic profile of the lens in a streptozotocin-induced diabetic rat model using shotgun proteomics
}

\author{
NORIAKI NAGAI ${ }^{1}$, TETSUSHI YAMAMOTO ${ }^{2}$, KUNIKO MITAMURA $^{2}$ and ATSUSHI TAGA ${ }^{2}$ \\ ${ }^{1}$ Department of Advanced Design for Pharmaceuticals; ${ }^{2}$ Pathological and Biomolecule Analyses Laboratory, \\ Faculty of Pharmacy, Kindai University, Higashi-Osaka, Osaka 577-8502, Japan
}

Received June 30, 2017; Accepted September 14, 2017

DOI: 10.3892/br.2017.988

\begin{abstract}
Streptozotocin (STZ)-induced diabetic rats (STZ rats) were used to investigate diabetic cataracts. In the current study, a shotgun liquid chromatography (LC)/mass spectrometry (MS)-based global proteomic analysis method was used to examine the mechanism of lens opacification as a result of hyperglycemia in STZ rats. The 6-week old Wistar rats were injected with STZ for 2 days $(100 \mathrm{mg} / \mathrm{kg} /$ day, i.p.) and housed for 3 weeks. The plasma glucose levels were identified to be significantly higher when compared with the normal rats and insulin was not detected in the STZ rats. Furthermore, opacification of the cortical epithelium was observed in the lenses of STZ rats. A total of 235 proteins were identified in the lenses of the STZ rats and 229 in the lenses of the normal rats. A label-free semi-quantitative method, based on spectral counting, identified 52 proteins that were differentially expressed in the lenses of STZ rats compared with normal rats. In particular, superoxide dismutase, which is a critical antioxidant enzyme that detoxifies superoxide through redox cycling, was downregulated when analyzed by the semi-quantitative method. In addition, phosphorylated-p38, which is important in the signaling pathway involved in the oxidative stress response, was significantly increased in the lenses of STZ rats when compared with normal rats $(\mathrm{P}<0.05)$. Thus, the changes in protein expression were evaluated in the lenses of STZ rats using a shotgun LC/MS-based global proteomic analysis approach, and a decrease in antioxidant enzymes and an increase in oxidative stress were identified in the lenses of
\end{abstract}

Correspondence to: Dr Atsushi Taga, Pathological and Biomolecule Analyses Laboratory, Faculty of Pharmacy, Kindai University, 3-4-1 Kowakae, Higashi-Osaka, Osaka 577-8502, Japan

E-mail:punk@phar.kindai.ac.jp

Abbreviations: LC/MS, liquid chromatography/mass spectroscopy; NSAF, normalized spectral abundance factor; ROS, reactive oxygen species; SOD, superoxide dismutase; STZ, streptozotocin

Key words: diabetic cataract, proteomics, lens, oxidative stress, streptozotocin-induced diabetic rats
STZ rats. Further studies are required to examine the role of these proteins in the onset or progression of diabetic cataracts.

\section{Introduction}

Diabetes mellitus is one of the most severe types of metabolic disorder in humans globally, and is characterized by insulin resistance and impaired insulin secretion (1). Long-term hyperglycemia in diabetics leads to many complications with tissues that require insulin for glucose entrance or with insulin-independent organs (2), and cataracts are one of the most common complications of exposure to uncontrolled chronic hyperglycemia in diabetes. It has been reported that the onset of cataracts in diabetic patients is 20 years earlier than in non-diabetic subjects (3).

Activation of the polyol pathway (4), non-enzymatic glycation of lens proteins (5-8) and increased oxidative stress (9-13) were reported as pathogenetic mechanisms of diabetic cataracts. In the polyol pathway, the excess glucose changes to sorbitol via aldose reductase and the excessive accumulation of sorbitol in the crystalline lens produces a high osmotic gradient, and causes the collapse and liquefaction of lens fibers, resulting in cataract formation $(14,15)$. Furthermore, enhanced osmotic stress leads to the production of reactive oxygen species (ROS) in the crystalline lens (16-18). In addition, sorbitol is metabolized to fructose. The fructose is metabolized into fructose-3-phospate and 3-deoxyglucosone (potent non-enzymatic glycation agents), which increase the quantity of advanced glycation end products leading to ROS generation $(19,20)$. Thus, numerous studies have evaluated diabetic cataracts and model animals have been used.

Streptozotocin (STZ)-induced hyperglycemia in experimental animals (STZ rats) has been widely used as a valuable model to investigate the effect of different hypoglycemic agents (21). The STZ damages pancreatic $\beta$-cells in rats, leading to deficient insulin secretion and a diabetic model $(22,23)$. The majority of diabetic rats are susceptible to the development of cataracts and lenticular polyol accumulation primarily induces cataractogenesis (14). However, all of the underlying mechanisms for lens opacification have not been elucidated in the STZ rats; therefore, further investigations are required. In the current study, a shotgun liquid chromatography (LC)/mass spectrometry (MS)-based global proteomic analysis of STZ rats was conducted to examine the underlying mechanism of 
lens opacification due to hyperglycemia. A total of 52 proteins were identified to be differentially expressed in the lenses of STZ rats compared with the lenses of normal rats, and these proteins may be involved in lens opacification.

\section{Materials and methods}

Materials. The following high-grade chemicals and reagents were purchased: Urea from GE Healthcare (Chicago, IL, USA) and thiourea from Nacalai Tesque, Inc. (Kyoto, Japan). All other chemicals and reagents were purchased from Wako Pure Chemical Industries, Ltd. (Osaka, Japan).

Animals. Healthy Male Wistar rats (mean weight, $220 \mathrm{~g} ; \mathrm{n}=10$ ) were provided from the Kiwa Laboratory Animals Co., Ltd. (Wakayama, Japan) and the 6-week old rats were injected with STZ for 2 days (100 mg/kg/day via i.p. injection). As lens opacification was initially observed 2-3 weeks after STZ treatment, the rats were evaluated 3 weeks after the injection of STZ to elucidate the early mechanism of diabetic cataracts. All of the experiments were performed in compliance with the regulations approved by the Ethics Committee of the Kindai University Faculty of Pharmacy (Osaka, Japan). The rats were housed in a room at $25^{\circ} \mathrm{C}$ under a 12-h light/dark cycle (2-3 rats/cage). All rats had access to food and water ad libitum.

Assay of glucose and insulin. Blood (50 ml) was sampled without anesthesia from the tail vein of each rat after fasting for $12 \mathrm{~h}$ (10:00 a.m.). The plasma glucose level was measured using an Accutrend GCT (Roche Diagnostics GmbH, Mannheim, Germany), and plasma insulin levels were assayed using an ELISA Insulin kit (cat. no. M1103; Morinaga Institute of Biological Science, Inc., Kanagawa, Japan) according to the manufacturer's protocol (24). The dynamic range of the ELISA Insulin kit is $0.1-6.4 \mathrm{ng} / \mathrm{ml}$.

Imaging of lens opacification in STZ rats. The rats were administered with $0.1 \%$ pivalephrine (Santen Pharmaceutical Co., Osaka, Japan) without anesthesia, and monitored using an EAS-1000 (Nidek Co., Ltd., Gamagori, Japan). The EAS-1000 conditions were as follows: Flash power index, 2,300 \pm 73 ; flash level, $100 \mathrm{~W} / \mathrm{sec}$; slit length and width, $5.0 \mathrm{~mm}$.

Protein extraction from the lenses of STZ rats. Three weeks after injection, the STZ rats were euthanized, and the lenses of the STZ rats were removed and homogenized in urea lysis buffer (7 M urea, $2 \mathrm{M}$ thiourea, 5\% CHAPS and $1 \%$ Triton $\mathrm{X}-100)$. The protein concentration was measured using a Bio-Rad Protein Assay kit (Bio-Rad Laboratories, Inc., Hercules, CA, USA).

In-solution trypsin digestion. A gel-free digestion approach was performed in accordance with the protocol described by Bluemlein and Ralser (25). In brief, $10 \mu \mathrm{g}$ protein extract from each sample was reduced by the addition of $45 \mathrm{mM}$ dithiothreitol and $20 \mathrm{mM}$ Tris(2-carboxyethyl)phosphine and alkylated using $100 \mathrm{mM}$ iodoacetic acid. Following alkylation, samples were digested with trypsin gold, MS grade (Promega Corporation, Madison, WI, USA) at $37^{\circ} \mathrm{C}$ for $24 \mathrm{~h}$. Subsequently, the digests were purified using PepClean C-18
Spin Columns (Thermo Fisher Scientific, Inc., Waltham, MA, USA) according to the manufacturer's protocol.

LC-MS/MS analysis for protein identification. Approximately $2 \mu \mathrm{g}$ peptide samples were injected onto a peptide L-trap column (Chemicals Evaluation and Research Institute, Tokyo, Japan) using an HTC PAL Autosampler (CTC Analytics AG, Zwingen, Switzerland) and further separated through a Paradigm MS4 (AMR, Inc., Tokyo, Japan) using a reverse-phase C18-column (L-column, gel particle diameter, $3 \mu \mathrm{m}$; $120 \AA$ A pore size, $0.2 \times 150 \mathrm{~mm}$; Chemicals Evaluation and Research Institute). The mobile phase consisted of solution $\mathrm{A}(0.1 \%$ formic acid in water) and solution B (acetonitrile). The column flow rate was $1 \mu \mathrm{l} / \mathrm{min}$ with a concentration gradient of acetonitrile, from 5\% B to $40 \% \mathrm{~B}$, for $120 \mathrm{~min}$. Gradient-eluted peptides were analyzed using an LTQ ion-trap mass spectrometer (Thermo Fisher Scientific, Inc.). The results were acquired in a data-dependent manner in which MS/MS fragmentation was performed on the two most intense peaks of every full MS scan.

All MS/MS spectral data were searched against the SwissProt Rattus Norvegicus database using Mascot (version_2.4.01; Matrix Science, London, UK). The search criteria were set as follows: Enzyme, trypsin; allowance of up to two missed cleavage peptides; mass tolerance $\pm 2.0 \mathrm{Da}$ and $\mathrm{MS} / \mathrm{MS}$ tolerance $\pm 0.8 \mathrm{Da}$; and modifications of cysteine carbamidomethylation and methionine oxidation.

Semi-quantitative analysis of identified proteins. The fold changes in expressed proteins, on a base-2 logarithmic scale, were calculated using the $\mathrm{R}_{\mathrm{SC}}$, based on spectral counting (26). Relative quantities of identified proteins were also calculated using the normalized spectral abundance factor (NSAF) (27). Differentially expressed proteins were selected when their $\mathrm{R}_{\mathrm{SC}}$ was $>1$ or $<-1$, which corresponded to fold changes of $>2$ or $<0.5$.

Western blot analysis. A total of $10 \mu \mathrm{g}$ lens extract was added to each well and subjected to $10 \%$ SDS-PAGE under reducing conditions, and the separated proteins were transferred to polyvinylidene fluoride membranes for $30 \mathrm{~min}$ at 15 V. Following blocking in Tris-buffered saline Tween-20 (TBST) buffer $(0.1 \%)$ with $5 \%$ skimmed milk for $2 \mathrm{~h}$ at room temperature, the membranes were incubated at $4{ }^{\circ} \mathrm{C}$ overnight with anti-phosphorylated (p)-p38 mitogen-activated protein kinase (MAPK; 1;1,000; cat. no. 4511) and anti-p38 MAPK (cat. no. 8690) antibodies (both from Cell Signaling Technology, Inc., Danvers, MA, USA), along with anti- $\beta$-actin antibody (cat. no. sc-47778; Santa Cruz Biotechnology, Inc., Dallas, TX, USA) to confirm equal loading of the proteins. The membranes were washed three times with TBST and incubated with horseradish peroxidase-conjugated anti-rabbit immunoglobulin G antibody (cat. no. A106PU; American Qualex, San Clemente, CA, USA) for $1 \mathrm{~h}$ at room temperature. Following washing, the blots were visualized using SuperSignal West Dura Extended Duration substrate (Thermo Fisher Scientific, Inc.) and bands were detected using a myECL Imager system (version 2.0; Thermo Fisher Scientific, Inc.). Subsequently, the intensity of p-p38 and p38 were quantified using myImage Analysis software (version 2.0; Thermo Fisher 
Scientific, Inc.) and the relative luminescence level of p-p38 over p38 was used to represent the signal strength of p-p38. All western blot analyses were performed in triplicate.

Statistical analysis. The unpaired Student's t-test was used and $\mathrm{P}<0.05$ was considered to indicate a statistically significant difference. All data are expressed as the standard error of the mean. The analyses were performed using GraphPad Prism software (version 5; GraphPad Software, Inc., La Jolla, CA, USA).

\section{Results}

Preparation of STZ-induced diabetic rats. Initially, the changes in plasma glucose and insulin were investigated in the rats following injection of STZ and whether the hyperglycemia caused lens opacification in the STZ rats was demonstrated. The plasma glucose levels in STZ rats $(247.1 \pm 10.6 \mathrm{mg} / \mathrm{dl} ; \mathrm{n}=5)$ were significantly higher than in the normal rats $(81.3 \pm 4.1 \mathrm{mg} / \mathrm{dl}$; $\mathrm{n}=5$ ). Furthermore, insulin was not detected in the STZ rats and the body weight in the STZ rats $(217.3 \pm 11.9 \mathrm{~g} ; \mathrm{n}=5)$ was significantly decreased when compared with that of the normal rats $(319.5 \pm 8.8 \mathrm{~g} ; \mathrm{n}=5)$. These results demonstrate that the STZ rats developed diabetes mellitus with hyperglycemia and hypoinsulinemia. In addition, opacification in the cortical epithelium was observed in the lenses of the STZ rats (Fig. 1).

Protein identification and profile of the lenses from STZ rats. To examine the effect of hyperglycemia on lens damage, the molecular profile of proteins whose expression level was changed in the diabetic rat model was investigated using shotgun proteomics. In the lenses of the STZ rats, 235 proteins were identified and 229 were identified in that of the normal rat (Normal) using the search parameters (Fig. 2). Among the 348 proteins identified in the rat lenses, $116(33.3 \%)$ were identified in the two groups; while, 119 (34.2\%) and 113 (32.5\%) proteins were unique to STZ and normal rats, respectively (Fig. 2).

Subsequently, a label-free semi-quantitative method based on spectral counting was used to identify proteins with expression levels regulated by hyperglycemia. The $\mathrm{R}_{\mathrm{SC}}$ value was plotted against the corresponding protein (x-axis) from left to right for proteins identified in the STZ and normal rats (Fig. 3). The positive and negative $R_{S C}$ values indicate increased and decreased expression levels, respectively in the STZ rats. The NSAF value was plotted against the corresponding protein. The NSAF of proteins in the STZ and normal rats are indicated above and below the $\mathrm{x}$-axis, respectively (Fig. 3). Proteins with either a high positive or negative $R_{S C}$ value were considered to be candidate proteins, whose expression was likely regulated by hyperglycemia. A total of 52 differentially expressed proteins were identified in the lenses of the STZ rats (Table I). The expression levels of housekeeping proteins, such as $\beta$-actin and glyceraldehyde-3-phosphate dehydrogenase, did not change in the lenses of the normal and STZ rats (Fig. 3).

Effect of hyperglycemia on the p38 signaling pathway in the lenses of STZ rats. To determine whether the oxidative stress response by active oxygen is affected by downregulation of superoxide dismutase (SOD) expression, the phosphorylation
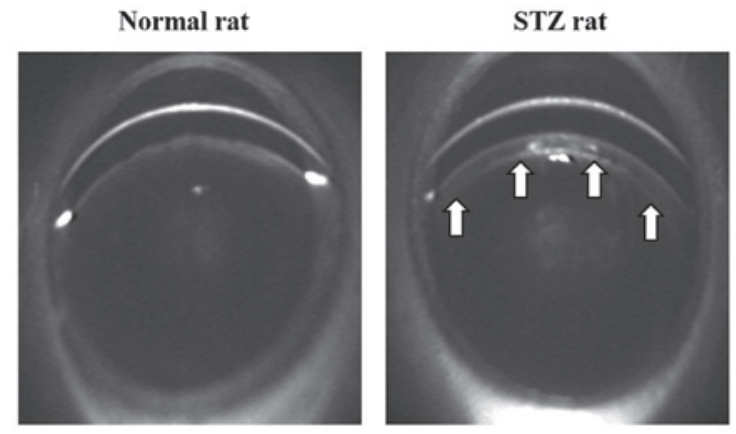

Figure 1. Scheimpflug slit images of lenses from normal rats and STZ-induced rats 3 weeks after injection of STZ. The images were obtained using an EAS-1000. Arrows indicate lens opacification. STZ, streptozotocin.

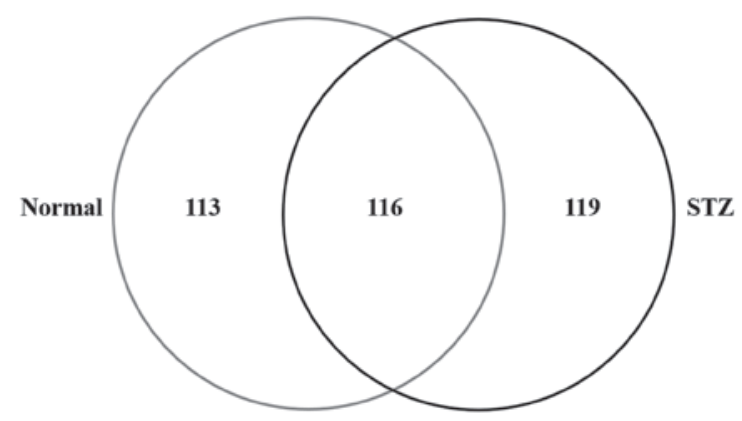

Figure 2. Venn map of proteins identified from the lenses of normal and STZ rats. There were 235 proteins identified in STZ-induced diabetic rats and 229 in the normal rats. STZ, streptozotocin.

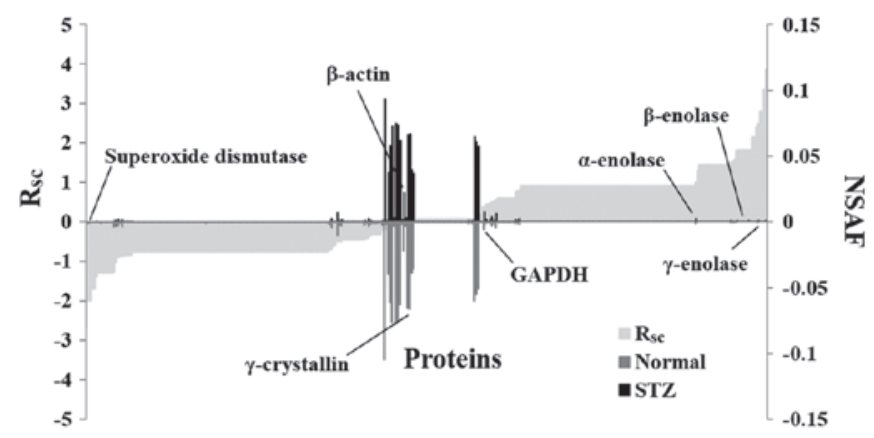

Figure 3. Semi-quantitative comparison of identified proteins in the lenses of STZ rats. The $\mathrm{R}_{\mathrm{SC}}$ and NSAF values were calculated for the proteins identified (x-axis), and the protein expression was compared between the STZ-induced diabetic rats and the normal rats. Proteins highly expressed in either the STZ or the normal rats are presented near the right or left side of the $\mathrm{x}$-axis. The housekeeping proteins are located around the center of the $\mathrm{X}$-axis. STZ, streptozotocin; NSAF, normalized spectral abundance factor.

of p38, which is important in the signaling pathways involved in oxidative stress response, were examined. The phosphorylation of $\mathrm{p} 38$ in the lenses of STZ rats was significantly increased when compared with the lenses of normal rats ( $\mathrm{P}<0.05$; Fig. 4).

\section{Discussion}

In the current study, a gel-free LC/MS-based proteomics approach was used to examine the effect of hyperglycemia on lens opacification. Although a quantitative value obtained using spectral counting may not be accurate (28), it is useful 
Table I. Differentially expressed proteins in the lenses of STZ rats.

\begin{tabular}{|c|c|c|c|c|c|c|}
\hline \multirow[b]{2}{*}{ No. } & \multirow[b]{2}{*}{ ID } & \multirow[b]{2}{*}{ Accession number and description } & \multirow[b]{2}{*}{$\begin{array}{l}\text { Amino } \\
\text { acids, } \mathrm{n}\end{array}$} & \multicolumn{3}{|c|}{ Spectral counting } \\
\hline & & & & Normal & STZ & $\begin{array}{l}\text { Fold change } \\
\left(\mathrm{R}_{\mathrm{SC}}\right)\end{array}$ \\
\hline 1 & SODC_RAT & P07632: Superoxide dismutase [Cu-Zn] & 154 & 4 & 0 & -1.995 \\
\hline 2 & HDAC1_RAT & Q4QQW4: Histone deacetylase 1 & 482 & 4 & 0 & -1.995 \\
\hline 3 & AK1CL_RAT & Q6AYQ2: Aldo-keto reductase family 1 member C21 & 318 & 3 & 0 & -1.690 \\
\hline 4 & LGSN_RAT & Q7TT51: Lengsin & 561 & 3 & 0 & -1.690 \\
\hline 5 & ARRS_RAT & P15887: S-arrestin & 403 & 5 & 1 & -1.398 \\
\hline 6 & TERA_RAT & P46462: Transitional endoplasmic reticulum ATPase & 806 & 2 & 0 & -1.302 \\
\hline 7 & ATIF1_RAT & Q03344: ATPase inhibitor, mitochondrial & 107 & 2 & 0 & -1.302 \\
\hline 8 & RHOB_RAT & P62747: Rho-related GTP-binding protein RhoB & 196 & 2 & 0 & -1.302 \\
\hline 9 & ENTP6_RAT & Q9ER31: Ectonucleoside triphosphate diphosphohydrolase 6 & 455 & 2 & 0 & -1.302 \\
\hline 10 & MYT1L_RAT & P70475: Myelin transcription factor 1-like protein & 1,187 & 2 & 0 & -1.302 \\
\hline 11 & RPGF2_RAT & F1M386: Rap guanine nucleotide exchange factor 2 & 1,496 & 2 & 0 & -1.302 \\
\hline 12 & LMIP_RAT & P54825: Lens fiber membrane intrinsic protein & 173 & 2 & 0 & -1.302 \\
\hline 13 & HS71L_RAT & P55063: Heat shock 70 kDa protein 1-like & 641 & 2 & 0 & -1.302 \\
\hline 14 & ACTC_RAT & P68035: Actin, $\alpha$ cardiac muscle 1 & 377 & 30 & 11 & -1.280 \\
\hline 15 & ACTG_RAT & P63259: Actin, cytoplasmic 2 & 375 & 25 & 11 & -1.027 \\
\hline 16 & ENOA_RAT & P04764: $\alpha$-enolase & 434 & 11 & 22 & 1.006 \\
\hline 17 & KCRB_RAT & P07335: Creatine kinase B-type & 381 & 1 & 4 & 1.301 \\
\hline 18 & K2C72_RAT & Q6IG04: Keratin, type II cytoskeletal 72 & 520 & 0 & 2 & 1.456 \\
\hline 19 & K1C14_RAT & Q6IFV1: Keratin, type I cytoskeletal 14 & 485 & 0 & 2 & 1.456 \\
\hline 20 & K1C13_RAT & Q6IFV4: Keratin, type I cytoskeletal 13 & 438 & 0 & 2 & 1.4563 \\
\hline 21 & PEBP1_RAT & P31044: Phosphatidylethanolamine-binding protein 1 & 187 & 0 & 2 & 1.456 \\
\hline 22 & COF1_RAT & P45592: Cofilin-1 & 166 & 0 & 2 & 1.456 \\
\hline 23 & NCAM1_RAT & P13596: Neural cell adhesion molecule 1 & 858 & 0 & 2 & 1.456 \\
\hline 24 & PGAM1_RAT & P25113: Phosphoglycerate mutase 1 & 254 & 0 & 2 & 1.456 \\
\hline 25 & PLEC_RAT & P30427: Plectin & 4,687 & 0 & 2 & 1.456 \\
\hline 26 & NMDE1_RAT & Q00959: Glutamate receptor ionotropic, NMDA 2A & 1,464 & 0 & 2 & 1.456 \\
\hline 27 & TCPG_RAT & Q6P502: T-complex protein 1 subunit $\gamma$ & 545 & 0 & 2 & 1.456 \\
\hline 28 & NPT2A_RAT & Q06496: Sodium-dependent phosphate transport protein 2A & 637 & 0 & 2 & 1.456 \\
\hline 29 & KIFC1_RAT & Q5XI63: Kinesin-like protein KIFC1 & 693 & 0 & 2 & 1.456 \\
\hline 30 & RL18_RAT & P12001: 60S ribosomal protein L18 & 188 & 0 & 2 & 1.456 \\
\hline 31 & COPG1_RAT & Q4AEF8: Coatomer subunit $\gamma-1$ & 874 & 0 & 2 & 1.456 \\
\hline 32 & HSP7C_RAT & P63018: Heat shock cognate $71 \mathrm{kDa}$ protein & 646 & 0 & 2 & 1.456 \\
\hline 33 & PDE3B_RAT & Q63085: cGMP-inhibited 3 ,5 -cyclic phosphodiesterase B & 1,108 & 0 & 2 & 1.456 \\
\hline 34 & K2C75_RAT & Q6IG05: Keratin, type II cytoskeletal 75 & 542 & 4 & 13 & 1.521 \\
\hline 35 & K2C4_RAT & Q6IG00: Keratin, type II cytoskeletal 4 & 536 & 1 & 5 & 1.552 \\
\hline 36 & ENOB_RAT & P15429: $\beta$-enolase & 434 & 2 & 8 & 1.588 \\
\hline 37 & TBA1C_RAT & Q6AYZ1: Tubulin $\alpha-1 C$ chain & 449 & 3 & 13 & 1.826 \\
\hline 38 & K2C8_RAT & Q10758: Keratin, type II cytoskeletal 8 & 483 & 0 & 3 & 1.844 \\
\hline 39 & K2C73_RAT & Q6IG03: Keratin, type II cytoskeletal 73 & 553 & 0 & 3 & 1.844 \\
\hline 40 & K2C7_RAT & Q6IG12: Keratin, type II cytoskeletal 7 & 457 & 0 & 3 & 1.844 \\
\hline 41 & ARF4_RAT & P61751: ADP-ribosylation factor 4 & 180 & 0 & 3 & 1.844 \\
\hline 42 & ARF1_RAT & P84079: ADP-ribosylation factor 1 & 181 & 0 & 3 & 1.844 \\
\hline 43 & TCAL7_RAT & D3ZT37: Transcription elongation factor A protein-like 7 & 98 & 0 & 3 & 1.844 \\
\hline 44 & TBB2B_RAT & Q3KRE8: Tubulin $\beta$-2B chain & 445 & 0 & 3 & 1.844 \\
\hline 45 & K1C15_RAT & Q6IFV3: Keratin, type I cytoskeletal 15 & 447 & 0 & 4 & 2.149 \\
\hline 46 & K1C17_RAT & Q6IFU8: Keratin, type I cytoskeletal 17 & 433 & 0 & 4 & 2.149 \\
\hline 47 & K2C1B_RAT & Q6IG01: Keratin, type II cytoskeletal 1b & 519 & 0 & 5 & 2.401 \\
\hline 48 & K2C5_RAT & Q6P6Q2: Keratin, type II cytoskeletal 5 & 576 & 2 & 16 & 2.490 \\
\hline 49 & ENOG_RAT & P07323: $\gamma$-enolase & 434 & 0 & 7 & 2.802 \\
\hline 50 & K1C10_RAT & Q6IFW6: Keratin, type I cytoskeletal 10 & 526 & 0 & 7 & 2.802 \\
\hline 51 & K2C6A_RAT & Q4FZU2: Keratin, type II cytoskeletal 6A & 552 & 0 & 11 & 3.374 \\
\hline 52 & K2C1_RAT & Q6IMF3: Keratin, type II cytoskeletal 1 & 625 & 0 & 16 & 3.869 \\
\hline
\end{tabular}

Expression levels of these 52 proteins were $>2$-fold higher or lower in the lenses of STZ rats compared with in the lenses of normal rats. STZ, streptozotocin. 


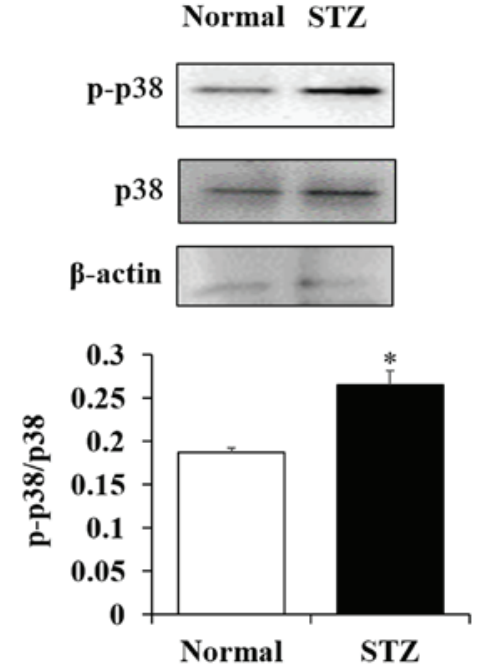

Figure 4. Effects of hyperglycemia on the p38 signaling pathway in the lens. The phosphorylation of p38 was significantly activated in the lenses of STZ-induced diabetic rats compared with in the lenses of normal rats. ${ }^{*} \mathrm{P}<0.05$. p-, phosphorylated; STZ, streptozotocin.

and has been used in previous studies investigating novel diagnostic biomarkers (29-34). Using semi-quantitative methods based on spectral counting, various proteins whose expression levels had changed by $>2$-fold were successfully identified in the lenses of STZ rats. Representative proteins involved in enzyme-associated glycolysis, such as $\alpha-, \beta$ - and $\gamma$-enolase, were upregulated by the injection of STZ. These enhanced enzymes may be induced by high glucose. Although Quinlan et al (35) reported that keratin was not found in the adult human lens, certain keratin proteins (type II cytoskeletal 4, 5 and 75) were detected in the lenses of normal rats. Furthermore, the expression levels of those keratin proteins in the lenses of STZ rats were enhanced when compared with those of normal rats. The expression levels of keratin proteins in lenses may differ between humans and rats. Therefore, this requires further investigation in future studies.

Additionally, SOD, which is a critical antioxidant enzyme that detoxifies superoxides via redox cycling and histone deacetylase 1 were downregulated. It is well known that chronic hyperglycemia increases the oxidant load (11) and leads to the onset of cataracts (12). Furthermore, the antioxidant capacity is reduced and the free radical load is increased in the eyes of diabetes mellitus patients. This change increases the susceptibility of the crystalline lens to oxidative damage (13). In addition, decrease in the antioxidant capacity is facilitated by advanced glycation and defects in antioxidant enzyme activity (13). Maurya et al (36) identified that the serum of SOD was significantly lower in patients with diabetic cataracts $(9.13 \mathrm{U} / \mathrm{ml})$ compared with patients with senile cataracts $(25.30 \mathrm{U} / \mathrm{ml})$ (36). These findings indicate that activation of the p38 signaling pathway, via downregulation of SOD expression, is one of the factors involved in the onset of lens opacification and that dysfunction of transcription, via low histone deacetylase 1, may be associated with the development of diabetic cataracts in STZ rats. Further studies are required to validate the results of spectral counting by western blotting and to elucidate the precise mechanisms for p38 in hyperglycemia-associated lens opacification using p38 inhibitors or corresponding knockout/knock-in strategies. In addition, it is important to validate these findings in human tissues. Therefore, our future studies will investigate the expression levels of SOD and p38 in human lens epithelial (HLE) SRA 01/04 cells exposed to high glucose conditions.

In conclusion, the changes in protein expression levels in the lenses of STZ rats were evaluated using a shotgun LC/MS-based global proteomic analysis, and an increase in oxidative stress in the lenses of STZ rats was observed. Therefore, oxidative stress may serve important roles in the progression of diabetic cataracts.

\section{Acknowledgements}

The present study was supported in part by a Grant-in-Aid for Scientific Research from the Japan Society for the Promotion of Science to T. Yamamoto (grant no. 15K09054).

\section{References}

1. Expert Committee on the Diagnosis and Classification of Diabetes Mellitus: Report of the expert committee on the diagnosis and classification of diabetes mellitus. Diabetes Care 26 (Suppl 1): S5-S20, 2003.

2. Brownlee M: Biochemistry and molecular cell biology of diabetic complications. Nature 414: 813-820, 2001

3. Hashim Z and Zarina S: Osmotic stress induced oxidative damage: Possible mechanism of cataract formation in diabetes. J Diabetes Complications 26: 275-279, 2012.

4. Srivastava SK, Ramana KV and Bhatnagar A: Role of aldose reductase and oxidative damage in diabetes and the consequent potential for therapeutic options. Endocr Rev 26: 380-392, 2005.

5. Ahmed N: Advanced glycation endproducts - role in pathology of diabetic complications. Diabetes Res Clin Pract 67: 3-21, 2005.

6. Araki N, Ueno N, Chakrabarti B, Morino Y and Horiuchi S: Immunochemical evidence for the presence of advanced glycation end products in human lens proteins and its positive correlation with aging. J Biol Chem 267: 10211-10214, 1992.

7. Duhaiman AS: Glycation of human lens proteins from diabetic and (nondiabetic) senile cataract patients. Glycoconj J 12: 618-621, 1995.

8. Lyons TJ, Silvestri G, Dunn JA, Dyer DG and Baynes JW: Role of glycation in modification of lens crystallins in diabetic and nondiabetic senile cataracts. Diabetes 40: 1010-1015, 1991.

9. Nagaraj RH, Sell DR, Prabhakaram M, Ortwerth BJ and Monnier VM: High correlation between pentosidine protein crosslinks and pigmentation implicates ascorbate oxidation in human lens senescence and cataractogenesis. Proc Natl Acad Sci USA 88: 10257-10261, 1991.

10. Shamsi FA, Sharkey E, Creighton D and Nagaraj RH: Maillard reactions in lens proteins: Methylglyoxal-mediated modifications in the rat lens. Exp Eye Res 70: 369-380, 2000.

11. Agte VV and Tarwadi KV: Combination of diabetes and cataract worsens the oxidative stress and micronutrient status in Indians. Nutrition 24: 617-624, 2008.

12. Jeganathan VS, Wang JJ and Wong TY: Ocular associations of diabetes other than diabetic retinopathy. Diabetes Care 31: 1905-1912, 2008

13. Ookawara T, Kawamura N, Kitagawa $\mathrm{Y}$ and Taniguchi N: Site-specific and random fragmentation of $\mathrm{Cu}, \mathrm{Zn}$-superoxide dismutase by glycation reaction. Implication of reactive oxygen species. J Biol Chem 267: 18505-18510, 1992.

14. Kinoshita JH: Mechanisms initiating cataract formation. Proctor Lecture. Invest Ophthalmol 13: 713-724, 1974.

15. Kinoshita JH: Cataracts in galactosemia. The Jonas S. Friedenwald Memorial Lecture. Invest Ophthalmol 4: 786-799, 1965.

16. Pollreisz A and Schmidt-Erfurth U: Diabetic cataract-pathogenesis, epidemiology and treatment. J Ophthalmol 2010: 608751, 2010.

17. Takamura Y, Sugimoto Y, Kubo E, Takahashi Y and Akagi Y: Immunohistochemical study of apoptosis of lens epithelial cells in human and diabetic rat cataracts. Jpn J Ophthalmol 45: $559-563,2001$. 
18. Li WC, Kuszak JR, Dunn K, Wang RR, Ma W, Wang GM Spector A, Leib M, Cotliar AM, Weiss M, et al: Lens epithelial cell apoptosis appears to be a common cellular basis for non-congenital cataract development in humans and animals. J Cell Biol 130: 169-181, 1995.

19. Tang WH, Martin KA and Hwa J: Aldose reductase, oxidative stress, and diabetic mellitus. Front Pharmacol 3: 87, 2012.

20. Stitt AW: The maillard reaction in eye diseases. Ann N Y Acad Sci 1043: 582-597, 2005.

21. Ivorra MD, Payá M and Villar A: A review of natural products and plants as potential antidiabetic drugs. J Ethnopharmacol 27: 243-275, 1989.

22. Stephen Irudayaraj S, Sunil C, Duraipandiyan V and Ignacimuthu S: Antidiabetic and antioxidant activities of Toddalia asiatica (L.) Lam. leaves in streptozotocin induced diabetic rats. J Ethnopharmacol 143: 515-523, 2012.

23. Nisha P and Mini S: Flavanoid rich ethyl acetate fraction of Musa paradisiaca inflorescence down-regulates the streptozotocin induced oxidative stress, hyperglycaemia and mRNA levels of selected inflammatory genes in rats. J Funct Foods 5: 1838-1847, 2013.

24. Nagai N, Ito $\mathrm{Y}$ and Sasaki H: Hyperglycemia enhances the production of amyloid $\beta 1-42$ in the lenses of Otsuka Long-Evans Tokushima fatty rats, a model of human type 2 diabetes. Invest Ophthalmol Vis Sci 57: 1408-1417, 2016.

25. Bluemlein $\mathrm{K}$ and Ralser M: Monitoring protein expression in whole-cell extracts by targeted label- and standard-free LC-MS/MS. Nat Protoc 6: 859-869, 2011.

26. Old WM, Meyer-Arendt K, Aveline-Wolf L, Pierce KG, Mendoza A, Sevinsky JR, Resing KA and Ahn NG: Comparison of label-free methods for quantifying human proteins by shotgun proteomics. Mol Cell Proteomics 4: 1487-1502, 2005.

27. Zybailov B, Coleman MK, Florens L and Washburn MP. Correlation of relative abundance ratios derived from peptide ion chromatograms and spectrum counting for quantitative proteomic analysis using stable isotope labeling. Anal Chem 77: 6218-6224, 2005.

28. Lundgren DH, Hwang SI, Wu L and Han DK: Role of spectral counting in quantitative proteomics. Expert Rev Proteomics 7: 39-53, 2010.
29. Yamamoto T, Kudo M, Peng WX and Naito Z: Analysis of protein expression regulated by lumican in PANC 1 cells using shotgun proteomics. Oncol Rep 30: 1609-1621, 2013.

30. Takaya A, Peng WX, Ishino K, Kudo M, Yamamoto T, Wada R, Takeshita $\mathrm{T}$ and Naito Z: Cystatin B as a potential diagnostic biomarker in ovarian clear cell carcinoma. Int J Oncol 46: 1573-1581, 2015.

31. Kanzaki A, Kudo M, Ansai S, Peng WX, Ishino K, Yamamoto T, Wada R, Fujii T, Teduka K, Kawahara K, et al: Insulin-like growth factor 2 mRNA-binding protein-3 as a marker for distinguishing between cutaneous squamous cell carcinoma and keratoacanthoma. Int J Oncol 48: 1007-1015, 2016.

32. Yamamoto T, Kudo M, Peng WX, Takata H, Takakura H, Teduka K, Fujii T, Mitamura K, Taga A, Uchida E and Naito Z: Identification of aldolase $\mathrm{A}$ as a potential diagnostic biomarker for colorectal cancer based on proteomic analysis using formalin-fixed paraffin-embedded tissue. Tumour Biol 37: 13595-13606, 2016.

33. Takata H, Kudo M, Yamamoto T, Ueda J, Ishino K, Peng WX Wada R, Taniai N, Yoshida H, Uchida E and Naito Z: Increased expression of PDIA3 and its association with cancer cell proliferation and poor prognosis in hepatocellular carcinoma. Oncol Lett 12: 4896-4904, 2016.

34. Kawamura T, Nomura M, Tojo H, Fujii K, Hamasaki H, Mikami S, Bando Y, Kato $\mathrm{H}$ and Nishimura T: Proteomic analysis of laser-microdissected paraffin-embedded tissues: (1) Stage-related protein candidates upon non-metastatic lung adenocarcinoma. J Proteomics 73: 1089-1099, 2010.

35. Quinlan RA, Sandilands A, Procter JE, Prescott AR, Hutcheson AM, Dahm R, Gribbon C, Wallace P and Carter JM: The eye lens cytoskeleton. Eye (Lond) 13 (Pt 3b): 409-416, 1999.

36. Maurya OP, Mohanty L, Bhaduri G and Chandra A: Role of anti-oxidant enzymes superoxide dismutase and catalase in the development of cataract: Study of serum levels in patients with senile and diabetic cataracts. J Indian Med Assoc 104: 394 , 396-397, 2006. 